\title{
Assessment of Conducted Emission for Multiple Compact Fluorescent Lamps in Various Grid Topology
}

\author{
Lok Choon Long ${ }^{1,2,3, *}$, Waseem El Sayed ${ }^{1,2,3}{ }^{\mathbb{D}}$, Venkatkumar Munesswaran ${ }^{1,2,3}$, Niek Moonen ${ }^{2}$ M, $^{\text {, }}$ \\ Robert Smolenski ${ }^{1}$ (D) and Piotr Lezynski ${ }^{1}$ (D)
}

1 Institute of Automatic Control, Electronics and Electrical Engineering, University of Zielona Gora, Licealna 9, 65-417 Zielona Gora, Poland; waseem.elsayed@ieee.org (W.E.S.);

Venkatkumar.Muneeswaran@nottingham.ac.uk (V.M.); r.smolenski@iee.uz.zgora.pl (R.S.); p.lezynski@iee.uz.zgora.pl (P.L.)

2 Faculty of Electrical Engineering, Mathematics and Computer Science, University of Twente, Drienerlolaan 5, 7522 NB Enschede, The Netherlands; niek.moonen@utwente.nl

3 George Green Institute for Electromagnetic Research, University of Nottingham, Nottingham NG7 2RD, UK

* Correspondence: c.l.lok@utwente.nl or c.lok@iee.uz.zgora.pl

check for

updates

Citation: Long, L.C.; Sayed, W.E.;

Munesswaran, V.; Moonen, N.;

Smolenski, R.; Lezynski, P.

Assessment of Conducted Emission for Multiple Compact Fluorescent Lamps in Various Grid Topology.

Electronics 2021, 10, 2258.

https://doi.org/10.3390/

electronics10182258

Academic Editor: Ahmed Abu-Siada

Received: 21 August 2021

Accepted: 10 September 2021

Published: 14 September 2021

Publisher's Note: MDPI stays neutral with regard to jurisdictional claims in published maps and institutional affiliations.

Copyright: (C) 2021 by the authors. Licensee MDPI, Basel, Switzerland. This article is an open access article distributed under the terms and conditions of the Creative Commons Attribution (CC BY) license (https:// creativecommons.org/licenses/by/ $4.0 /)$.

\begin{abstract}
This paper presents the measurement of aggregated conducted emission in the frequency range of $9 \mathrm{kHz}$ to $150 \mathrm{kHz}$ produced by multiple compact fluorescent lamps (CFL) and how it equates to a multiple power converter system. Discrepancies in peak emission measurement results related to this application are illustrated to understand the underlying issue related to volatility of frequency components. Furthermore, this knowledge analyzes the relation of electromagnetic disturbances with respect to different topological network connections. The final presented results constitute theoretical description and statistical information about the characteristics of conducted emission measured in this multi-converter system.
\end{abstract}

Keywords: CFL; conducted emission; DM; EMI; grid topology; power converter

\section{Introduction}

Nowadays, power converters are used in the majority of household appliances due to the benefits with respect to volume, weight, performance, and energy efficiency. The power converters used in residential appliances generally have switching frequencies from a few $\mathrm{kHz}$ to tens of $\mathrm{kHz}$, these appliances can generate a substantial amount of electromagnetic interference (EMI) in that frequency range. In particular, power converter technology has been widely implemented in lighting application since the introduction of Regulation (EC) Number 244/2009 and 245/2009, which were introduced to phase out the incandescent lamps [1,2]. Consequently, the usage of fluorescent lamps (FL) being used in many households and its usage is likely to increase in the future [3]. For instance, in Organisation for Economic Co-operation and Development (OECD) countries, the demand for FLs has risen significantly over the last decades and continues to rise from an estimated 1.2 billion in 2020 to 1.6 billion in 2027 [4]. Moreover, there are local regulatory guidelines that specify illuminance in workspace. For instance, a standard indoor workspace requires 300 to 500 lumen per square-meter illumination to meet the desired requirement $[5,6]$. Hence, a higher number of lamps per floor area is used. With large number of lamps connected in electrical grid, their combined impact to the electrical grid is not negligible. Meanwhile, the effects of power quality and harmonic have been the subject of research for many years, with a summary and discussion of ongoing areas of research available in [7-11]. Those research studies are mainly focus on emphasize dedicated to voltage and current distortion compliance evaluation. As such, there is little evaluation on the grid configuration for measurements for household appliances such as the CFL lamps.

The EU directive states that the equipment intended to be connected to network should prevent networks from suffering degradation under normal condition [12], despite 
the evaluation of cumulative effect have been shown in [11], where it was mention that there are no details concerning the type of network used for the EMC evaluation process. Network configuration can play an important role in the assessment of electromagnetic interference (EMI) as the equipment emissions aside from the sources of EMI itself. For example, the conducted EMI injected into the supply network, can affect the network impedance due to the interaction among the loads [13-15]. This happens whether the type of appliance that produces EMI signals with small frequency difference or large frequency difference can decrease or increase the total conducted EMI in the network, whose these spurious conducted EMI contents have also been identified as a cause of unreliable measurement outcome [11,16-19]. Moreover, this situation can be complicated dealing with highly volatile and random-modulated EMI contents produced by multiple power converters. Therefore, without a clear understanding of the interactions between grid configuration and underlying EMI contents, it is difficult to properly justify whether the type of grid configuration consideration can be completely omitted from a standard EMC assessment.

In this paper, comparative analysis concerning aggregated EMI generated by a series of CFL lamps in several network topologies are compared and studied. The number of lamps was varied to investigate the micro-effect in terms of aggregated EMI in different topologies. The goal of this work is to understand the aggregated EMI originating from multiple similar sources that are interconnected in different topologies. The emissions are systematically analysed in order to highlight the measurement challenges and their impact on the EMC assessment. Thus, to be able to better understand issue concerning EMC measures related to this application. This paper is organized in this order. In Section 2, the illustration of high volatility EMI signals process and the mathematical characterization are introduced. In Section 3, the measurement setup is discussed. In Section 4, measurement results are presented and discussed to verify the measurement issue. Finally, conclusion in Section 5 .

\section{Processing Highly Volatile Sinusoidal Components in EMI Receiver}

EMI exists in the form of unsteady, impulsive noise which can be attributed as narrowband or broadband signal. They are difficult to be measured due to unpredictable leakage path in an electrical environment, but possible when different detectors can be used to synthesize the time-domain signals. These detectors are called the peak, quasi-peak, average and RMS (root mean square) detector which feature the fundamental element in a modern real-time EMI receiver. An EMI receiver is basically a super heterodyne receiver with frequency-selective amplifier [20], it can be divided into four parts: preselection stage, mixer, intermediate frequency (IF) bandpass filter and amplifier stage, and detector stage as shown in Figure 1.

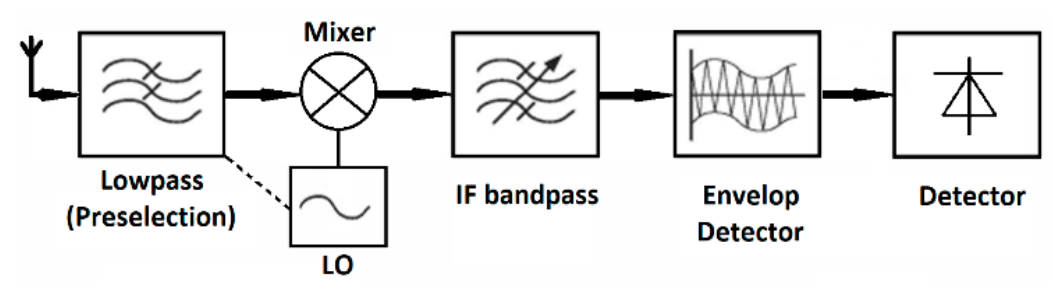

Figure 1. Model of an EMI receiver.

Currently, the working principle of EMI receiver can be emulated in computer-based programming software such as Matlab ${ }^{\circledR}$ and Python ${ }^{\circledR}$, this is made possible with the fast Fourier transform (FFT) and short-time Fourier transform (STFT) functions [20,21]. For instance, a low-pass filter can be programmed to present the preselection stage, a voltage control oscillator (VCO) function is used as mixer to shift frequency to intermediate frequency, the selective/bandpass IF filter is a Gauss-like function pattern that extracts 
fragment of the signals into multiple frequency windows. Precise descriptions of such filters is given in [22]. The last stage is the envelope detector for evaluating the filtered signals.

According to the international electrotechnical terminology, a narrowband disturbance is "an electromagnetic disturbance, or component thereof, which has a bandwidth less than or equal to that of a particular measuring apparatus, receiver or susceptible device". On the other hand, a broadband disturbance is defined as "an electromagnetic disturbance which has a bandwidth greater than that of a particular measuring apparatus, receiver or susceptible device". Hence, the signal classification whether a broadband or narrowband type is determined by the frequency spectrum of the signal under study, it is related to the intermediate frequency bandwidth (IFBW) of the instrument used for measurement. For instance, shown in Figure 2, a signal spectrum that is bound within the passband of the IF filter, it is a narrowband signal otherwise it is the broadband signal. In response to this, the IFBW selection is one essential factor for emulating the EMI receiver accurately. Details about standardized EMC measurement has been elaborated in [12,23].

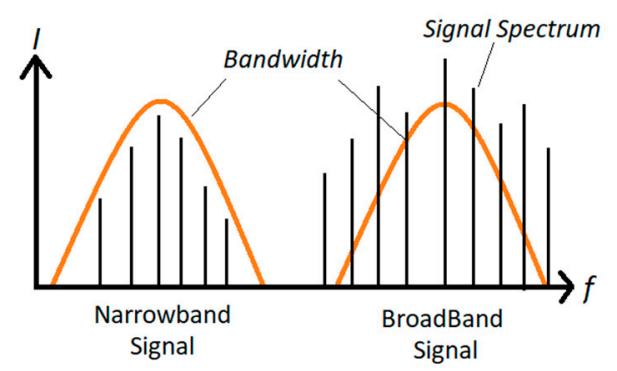

Figure 2. Generic definition of narrowband and broadband signals with spectrum measurement via an EMI receiver.

Figure $3 \mathrm{~b}$ shows the representation of high frequency output signal of a compact fluorescent lamp in time-domain, it consists of different frequency in each oscillating cycle inversely proportional to its amplitude, and Figure $3 \mathrm{~d}$ is the measured conducted emission in frequency-domain. Mathematically, this signal intensity, I for one sinusoidal component in time domain can be represented by:

$$
I(t)=\sum_{n=1}^{\infty} A_{n} e^{t / \tau}\left[\sin \left(2 \pi\left(f_{n}-t\right) t+\theta\right)\right]
$$

where $\tau=\frac{1}{2 \pi f_{n}} . A_{n} e^{t / \tau}$ is the amplitude of each wave cycle corresponding to its frequency, $f_{n}$. In a multiple CFL lamps setup, aggregation of these signals tends to be more complex given their amplitudes are not the same periodically [24]. Let assume their phase different, $\theta$ is zero and both $A_{1}$ and $A_{2}$ are same at unity. The instantaneous value can be obtained via expression:

$$
\begin{array}{r}
I\left(t ;\left\{f_{1}, f_{2}\right\}\right)=A_{1} e^{\frac{t}{\tau}} \cdot \sin \left(2 \pi\left(f_{1}-t\right) t\right)+A_{2} e^{\frac{t}{\tau}} \cdot \sin \left(2 \pi\left(f_{2}-t\right) t\right) \\
\quad=2 e^{t / \tau}\left[\sin \left(2 \pi\left(\frac{f_{1}+f_{2}}{2}-t\right) t\right) \cos \left(2 \pi\left(\frac{f_{1}-f_{2}}{2}-t\right) t\right)\right]
\end{array}
$$

According to superposition theory, aggregating two or more sinusoidal components can result in envelope formation similar to the amplitude modulation (AM) phenomenon given that $\max \left|\frac{f_{i}-f_{j}}{2}\right| \leq \min \left|\frac{f_{i}+f_{j}}{2}\right|$ [24]. The value of the envelope can be obtained using:

$$
S_{\text {env }}\left(t,\left\{f_{1}, \ldots, f_{n}\right\}\right)=\sum_{\substack{2 \ll i \ll n \\ 1 \ll j<i}}^{n}\left[\frac{2 e^{t / \tau}}{n-1}\right] \cdot\left[\sin \left(2 \pi\left(\frac{f_{i}+f_{j}}{2}-t\right) t\right) \cos \left(2 \pi\left(\frac{f_{i}-f_{j}}{2}-t\right) t\right)\right]
$$


where $\left[\frac{n e^{-t / \tau}}{n-1}\right]$ is the resultant amplitude with respect to the number of signals, $\mathrm{n}$. Now, signal in the form of envelope is fed into EMI receiver input. After mixer stage, the selected signal is swept by IF filter $[25,26]$. Hence, the instantaneous time domain output signal of the IF filter seen by an EMI receiver can be formulated as:

$I_{f}(t)=\sum_{f_{k}-\frac{f_{I F}}{2}}^{f_{k}+\frac{f_{I F}}{2}} H\left(e^{j 2 \pi f_{k}}\right) \sum_{\substack{2 \ll i \ll n \\ 1 \ll j<i}}^{n}\left[\frac{2 e^{t / \tau}}{n-1}\right] \cdot\left[\sin \left(2 \pi\left(f_{I F}-f_{k}+f_{A}-t\right) t+\theta\right) \cos \left(2 \pi\left(f_{I F}-f_{k}+f_{B}-t\right) t\right)+\theta\right]$

where $f_{A}=\left(\frac{f_{i}+f_{j}}{2}\right)$ and $f_{B}=\left(\frac{f_{i}-f_{j}}{2}\right)$ are the maximum and minimum beating frequencies of oscillatory envelope, $f_{k}$ is the selected filter frequency, $f_{I F}$ is the bandwidth of the IF filter, $H\left(e^{j 2 \pi f}\right)$ is the transfer function of the IF filter at envelope input frequency. From expression (4), it indicates that the output is the product of envelope and frequency response of the IF windows function filter. The frequency responses of IF bandpass and window function filters can be found in [25]. Further details about detector models have been discussed in [27-29]. Hence, is not covered in this paper.

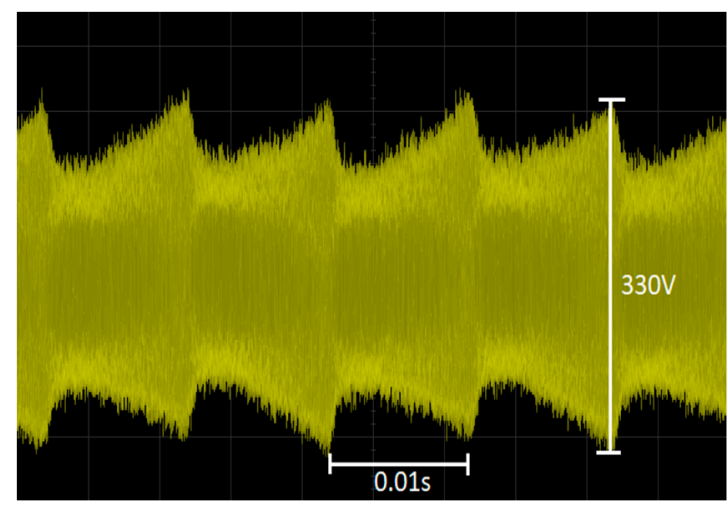

(a)

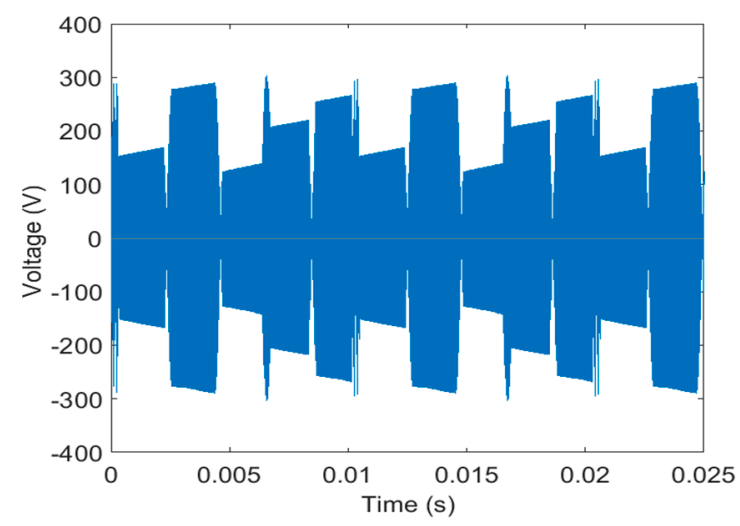

(c)

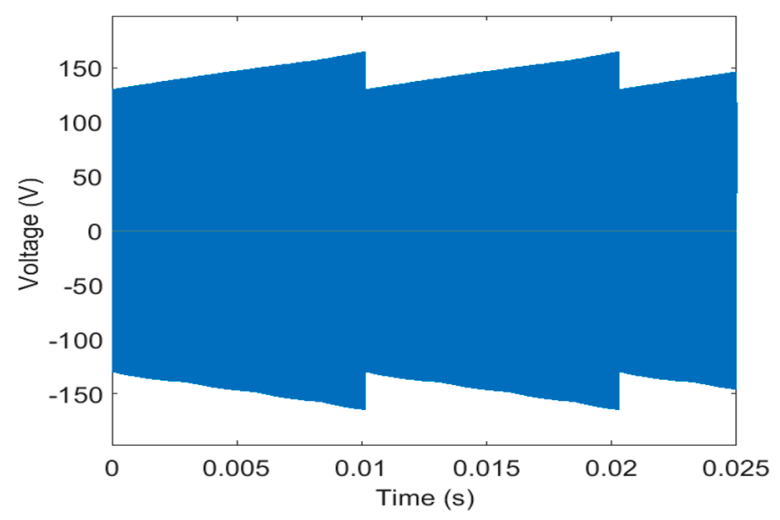

(b)

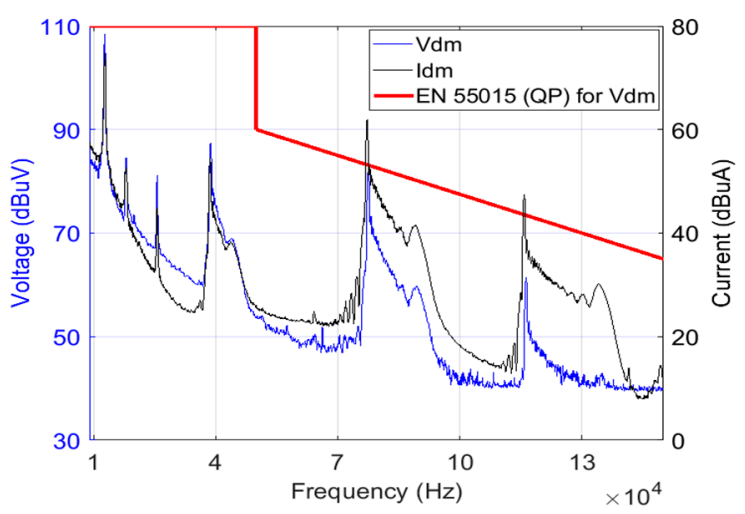

(d)

Figure 3. (a) Measured high frequency output signal of fluorescent lamp in time-domain. (b) Representation for high frequency output signal of fluorescent lamp in time-domain. (c) Envelope formation in time domain by aggregating two random signals depicted in (b). (d) Power spectrum density (PSD) output in frequency-domain.

EMI in CFL lamps, however, consist of highly volatile and transient signals. In order to illustrate the exact spectrum of these sinusoidal components captured by an EMI receiver, a spectrogram is generated in Matlab ${ }^{\circledR}$ modelled with a Kaiser window function, the IF filter is modelled according to its impulse bandwidth, noise-bandwidth, and the filter masks, as 
defined in CISPR 16-1-1. This spectrogram shows a discretization in frequency and time. The resolution in frequency is described by the frequency bin width $\Delta \mathrm{f}$ shown in Figure 4 . However, with sufficient number of overlapping frequency bins, the amplitude error can be negligible hence is not considered in this illustration [28]. The STFT processes input signal with fluctuating individual frequency in the range $40 \mathrm{kHz}$ to $50 \mathrm{kHz}$, the discretization in the time domain has sampling frequency of $1 \mathrm{MHz}$, a 0.8 overlapping factor was used to achieve a sufficiently dense discretization for this simulation. For the aggregation, with convoluted Gaussian-like windows discussed above, the IFBW was set to $200 \mathrm{~Hz}$, which correspond to parameters required in [22,29].

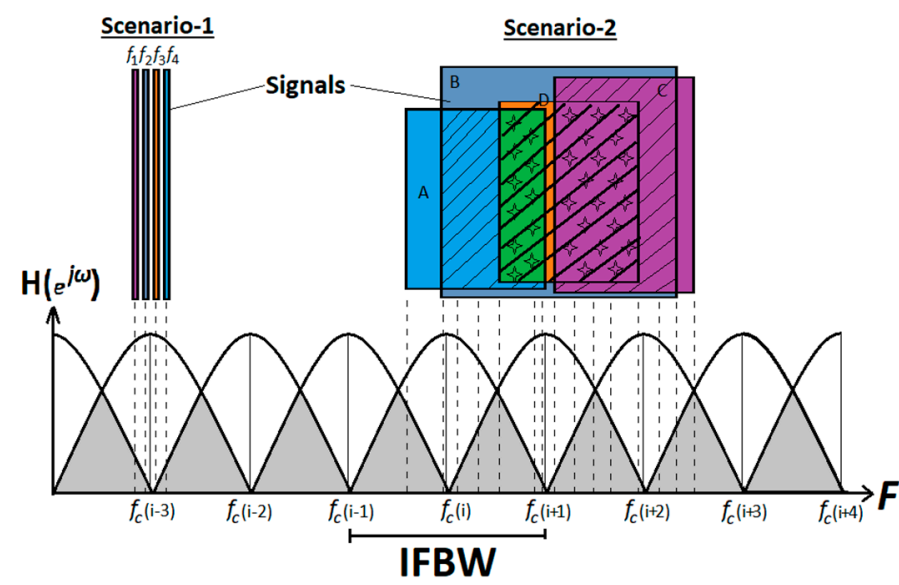

Figure 4. Operation diagram for IF bandpass filtering of narrowband and broadband signals: Scenario-1: no signal overlap. Scenario-2: signals overlapped.

In Figure 4, the picture shows 4 signals swept by many gapless IF Gaussian windows. The higher percentage of these windows overlapping, the higher the accuracy of single impulses measurement during the dwell time. There are two scenarios depicted, a narrowband signal in Scenario-1 consists of four absolute frequency components with slightly different frequencies bound within an IFBW, an envelope is formed due to their interaction, the output will be one aggregated discrete spectrum as the result from superposition of multiple sinusoidal components [11]. Therefore, the peak total emission increases proportionally with the number of signals. In Scenario-2, the signals are sinusoidal components with randomly changing frequencies within a large bandwidth, the frequency variation is very large and spontaneous, there are regions (diagonal lines) where superposition occurs among these sinusoidal components randomly. As a result, measuring these highly volatile signals can produce inconsistent peak total emission with respect to the number of signals.

Figure 5 shows the 3D spectrogram of aggregated signal corresponds to frequency response of the Kaiser IF filter convolved (overlap) with discrete frequency bins. Apparently, the mean total peak emission is not same in both cases despite the number of signals is equal. As we can see in Figure 5a, aggregating 5 sinusoidal components with small frequency variation results in constant peak emission. On the other hand, as shown in Figure $5 \mathrm{~b}$, high volatility sinusoidal components with large frequency variation sweep across many frequencies bin in a wide bandwidth, the peak value of envelope changes rapidly at every instant of time. As a result, the instantaneous peak emission scatters along the time-axis, which is indicated by red marks, the energy is spread into wide spectrum band compared to narrow spectrum in Figure 5 a despite equal power delivered by these signals. By these examples, it is obvious to note that increasing the number of signals does not necessarily increase its peak total emission in a network. 


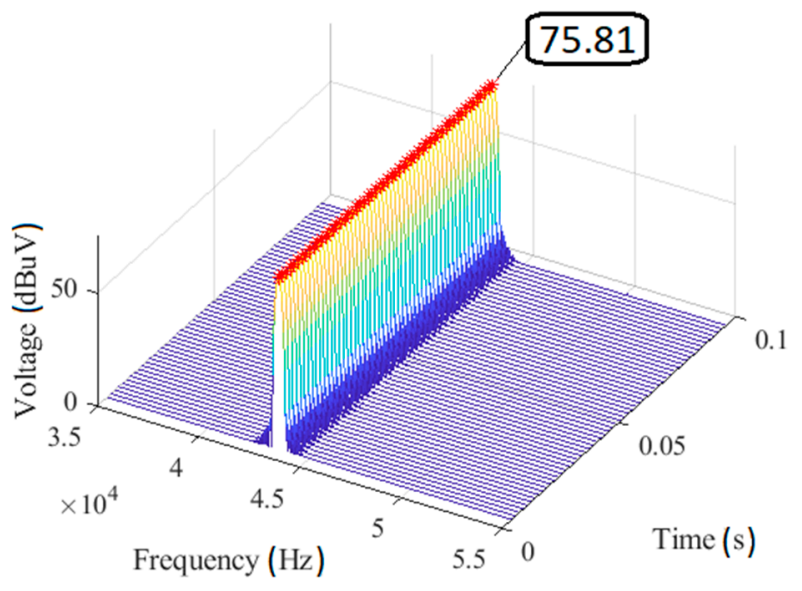

(a)

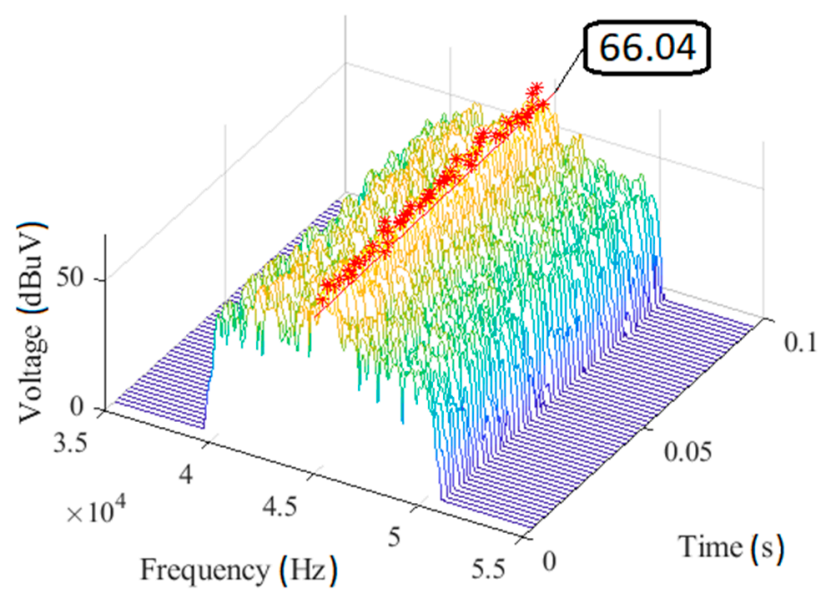

(b)

Figure 5. The 3D waterfall spectrogram of aggregated signal generated by five $45 \mathrm{kHz}$ sinusoidal components with various frequency difference range: (a) $\pm 20 \mathrm{~Hz}$. (b) $\pm 5 \mathrm{kHz}$.

A standard time domain EMI receiver model works diligently dealing with small frequency variation signals, however, rapid frequency changes in signal larger than the IFBW during measurement can be a reason why the level of measured peak total emission is disproportional to the number of injected signal $[18,30]$. Analogously, a paper published by Piotr et al. has presented that a standard IFBW for CISPR A is usually too narrow for proper evaluation of random modulated signal, which frequency varies in a wider range. For a higher frequency range (CISPR-B) the measured difference between randomly modulated (RM) signal and deterministic modulated signal is insignificant [31].

In regard to that, preliminary measurements concern aggregated signals were conducted using the National Instrument ${ }^{\circledR}$ FPGA control coupled to an EMI receiver in order to verify that the described issue is valid, the schematic of test setup diagram is shown in Figure 6 and results are summarized in Figure 7. The box-and-whisker plots presented show the distribution of one-thousand peak values measurement for each change of the number of lamps. For ease of measurement output comparable to previous related articles such as [11,19], each measurement has been made with the average (AV) detector in the EMI receiver and data are normalized to time step of $1 \mathrm{~s}$ in our analysis. Our measurement results indicate that mean emission values for aggregating similar frequency or slightly different frequency of sinusoidal components increase with the number of signals. In the presented case it is about $110 \mathrm{mV}$ or $100.8 \mathrm{dBuV}$ increase between 1 and 5 signals. Meanwhile, it is obvious that the mean emission values decrease in the case for high volatility sinusoidal components with large frequency variation, the largest drop of signals is between 1 and 5 signals. Subsequently, the mean emission values decrease until 15 signals. Therefore, these results have clearly indicated discrepancy in measurement for mean peak emission although the number of signals is similar. Due to such measurement outcome, it might be interesting on how the level of the spectrum is impacted based on different grid topology with respect to the number of signals. In the following section, we look into investigation whether different topology will also observe similar underlying trend in measurement outcome.

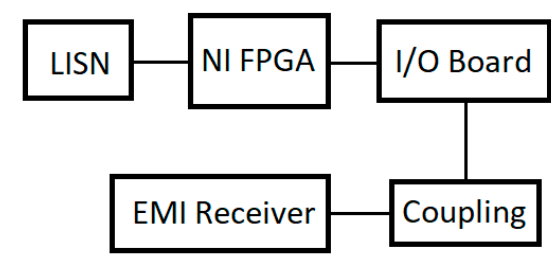

Figure 6. The testing scheme for measuring the total aggregated signals. 


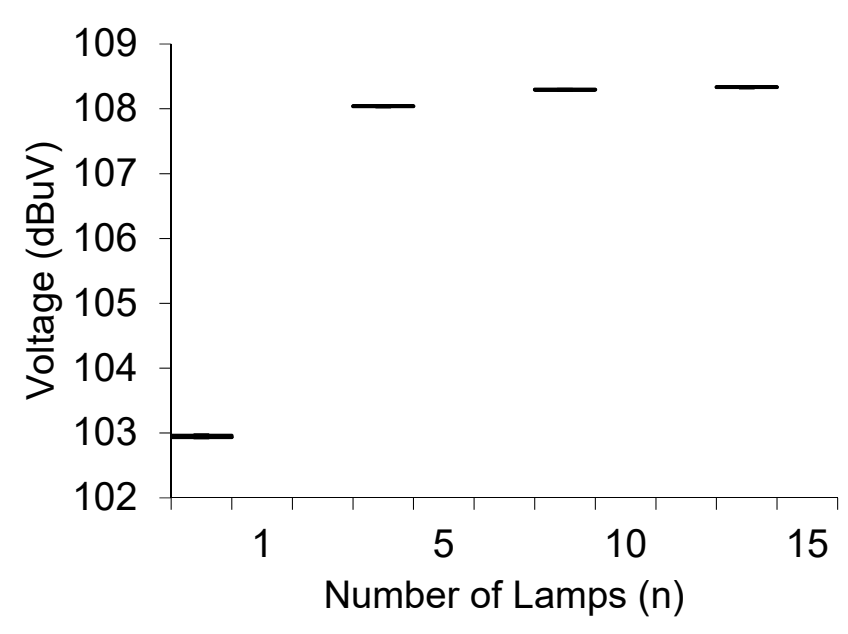

(a)

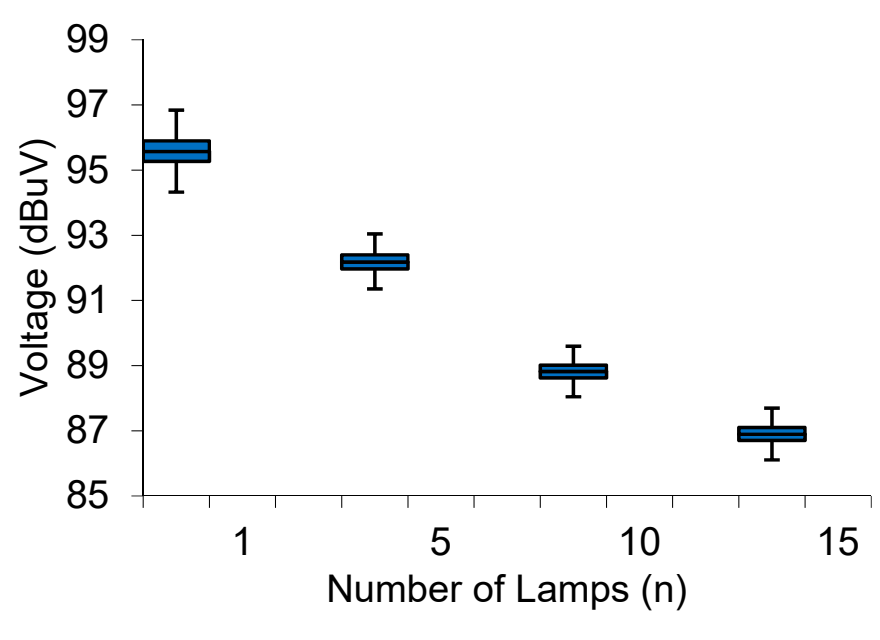

(b)

Figure 7. Box-and-whisky plots of conducted emission by AV detector with $200 \mathrm{~Hz}$ IFBW at switching frequency $20 \mathrm{kHz}$ with frequency variation. (a) $\pm 0.01 \mathrm{~Hz}$. (b) $\pm 2500 \mathrm{~Hz}$.

\section{Measurement Setup}

The measurement considered four standard CFLs rated $230 \mathrm{~V}, 50 \mathrm{~Hz}$. The four CFL lamps with a wired network are the equipment-under-test (EUT). The lamp used is the Philips $20 \mathrm{~W}$ CFL at $\mathrm{PF}=0.6$. In contrast to most power converters where their switching frequency is fixed which is driven by high precision crystal oscillator with negligible frequency tolerances. However, the output for the gas discharge type CFL lamp operates at unfixed resonant switching frequency [32,33], the observed operating switching frequency range is between $37 \mathrm{kHz}$ to $47 \mathrm{kHz}$ for this lamp, a line impedance stabilization network (LISN) is used for isolating the test setup from the interference outside of the setup and provide constant impedance during the measurement. The differential mode (DM) signals were measured using a Gauss ${ }^{\mathrm{TM}}$ TDEMI receiver that is connected to an in-situ EMI monitoring device (MD) developed by [29,34]. Each bulb is separated by $2 \mathrm{~m}$ to mimic the standard indoor ceiling lighting point distance. The experiment was conducted with no cable overlap to avoid any cross-talk effect as shown in Figure 8. The lamps were being turned on follows the number sequence indicated in Figure 9.

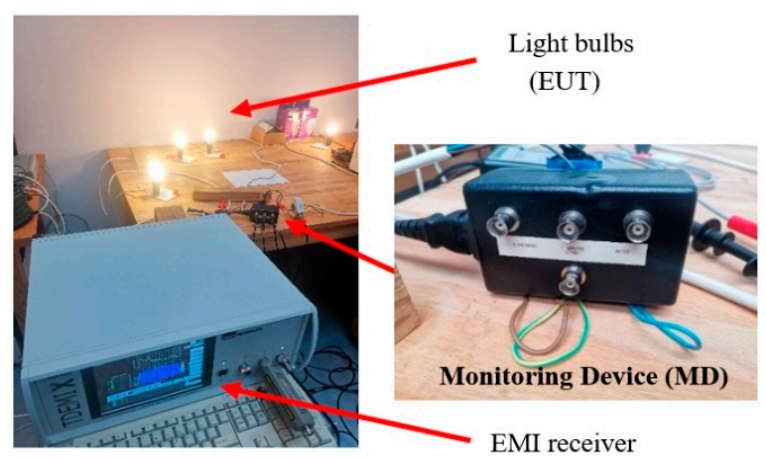

Figure 8. Measurement setup.

Since this research is carried out with the view to assess the aggregated EMI in CFL lamps interconnected in different topological networks, the configurations are illustrated below. 


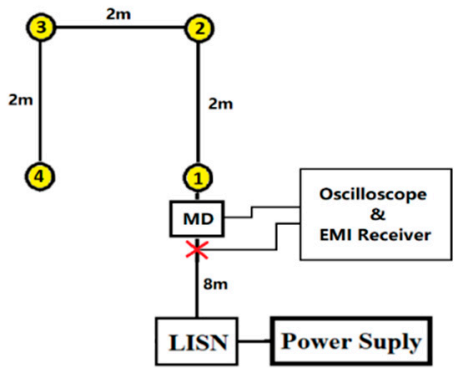

(a)

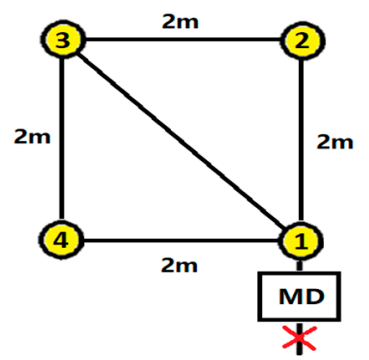

(c)

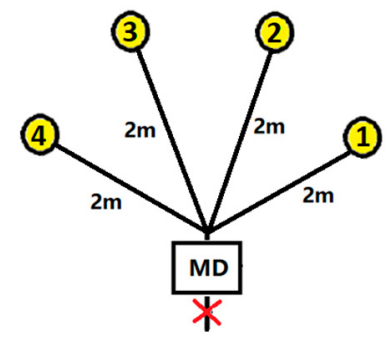

(e)

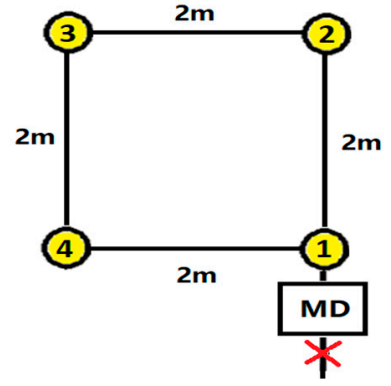

(b)

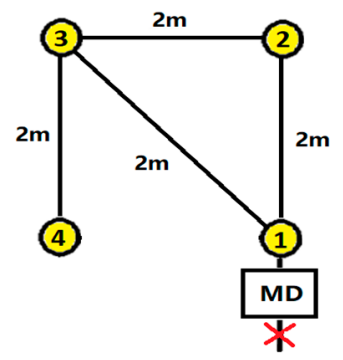

(d)

Figure 9. Single-line-diagram of CFL lamps for various grid topology. (a) Type-A connection. (b) Type-B connection. (c) Type-C connection. (d) Type-D connection. (e) Type-E connection.

\section{Experimental Results and Discussion}

Experiments for standardized EMI measurements have been conducted in laboratory setup compliant with CISPR-16-1-1 and EN 55015 [20]. The measurement for the EUT under steady state have been recorded and were later processed using Matlab ${ }^{\circledR}$. The voltage $(V)$ and current $(I)$ values each in a period of $1000 \mathrm{~s}$ with dwell time 100 milliseconds and IFBW $=200 \mathrm{~Hz}$ were being recorded. The EMI receiver provided the spectrogram and PSD for both quasi peak (QP) and AV detector. Since the operation of lamps operates at nonconstant switching frequency. Hence, this characteristic affects the impedance hence the voltage stability of the network that it is connected to because the impedance is the function of frequency, $2 \pi f$ which is determined by the real value resistance, $R$ and capacitive, $C$ in its parallel-resonant circuit [32,33]. These resonant converters in these CFL lamps connected in same network can cause the impedance of the grid to continuously fluctuate.

Figure 10 illustrates the measurement results for the DM values in terms of $\mathrm{dB} \mu \mathrm{V}$ and $\mathrm{dB} \mu \mathrm{A}$ which are derived from the peak values at about $40 \mathrm{kHz}$ comparable to results shown in Figure 5b. Similarly, the box-and-whisker plots presented show the distribution of one-thousand peak values measurement for each changes of the number of lamps. Each measurement has been made according to aforementioned general practice via the AV detector and data are normalized to time step of $1 \mathrm{~s}$ for analysis $[11,19,31]$. 


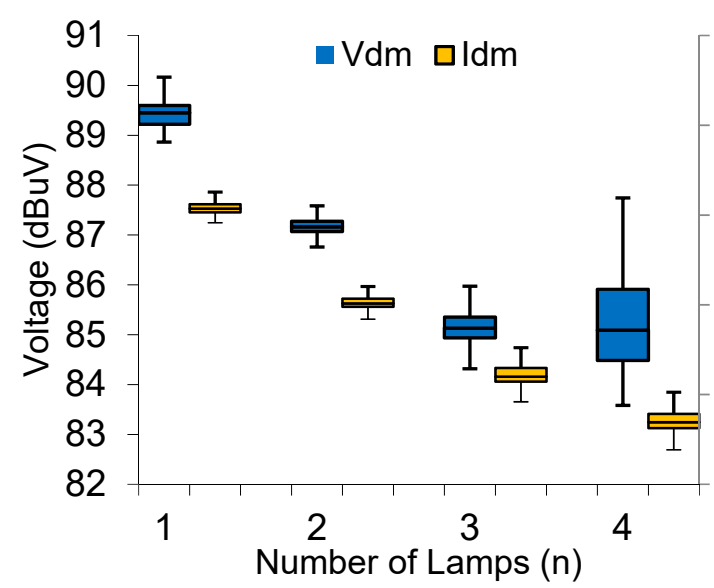

(a)

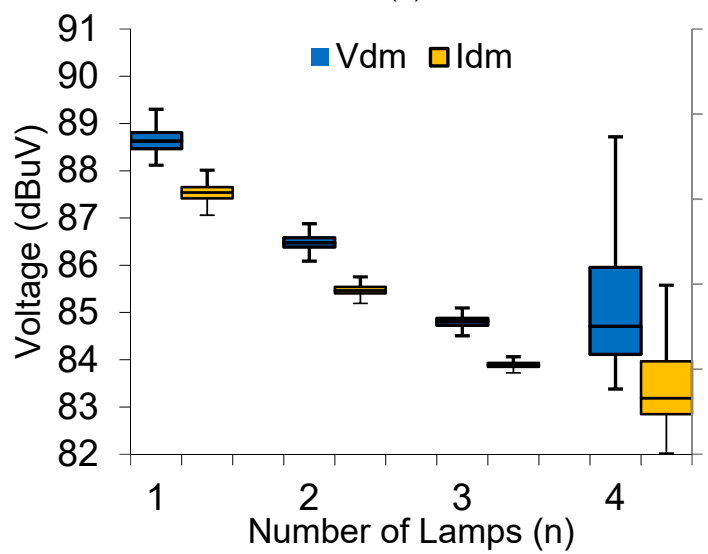

(c)

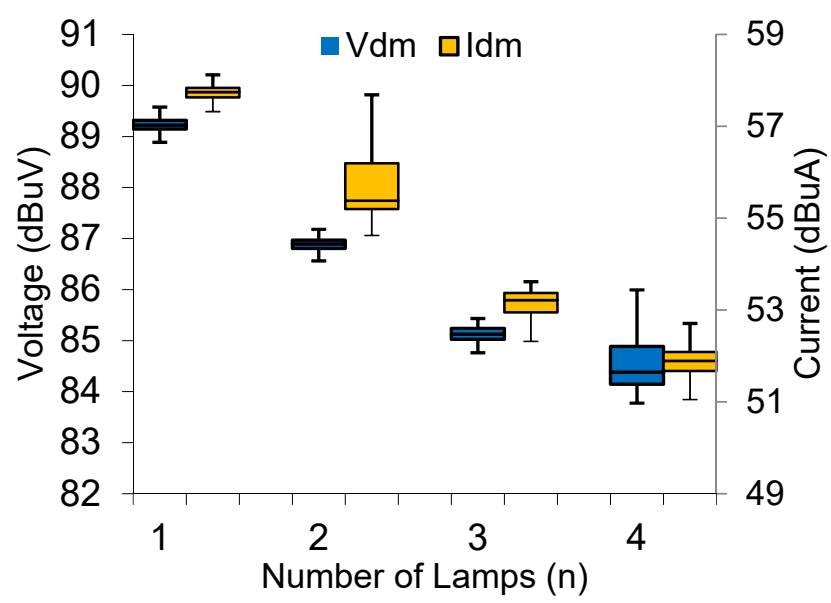

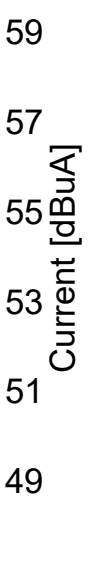

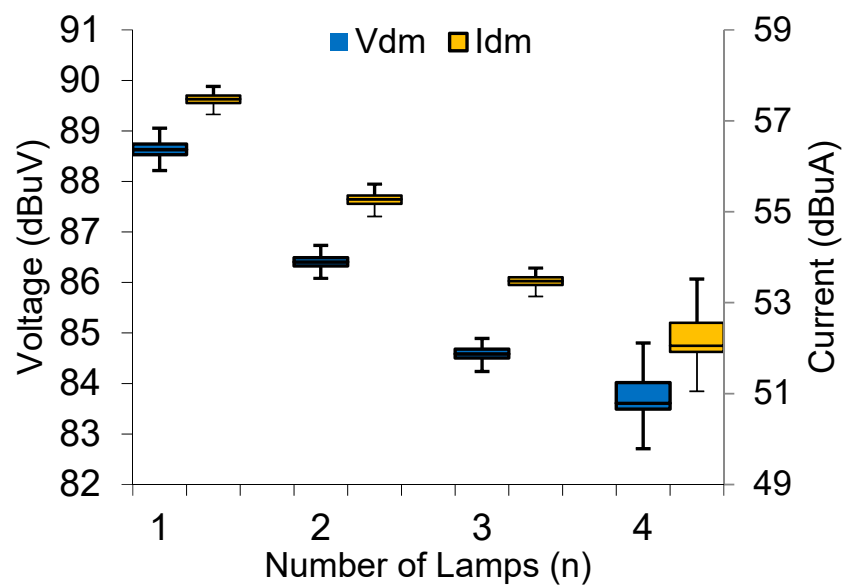

(b)
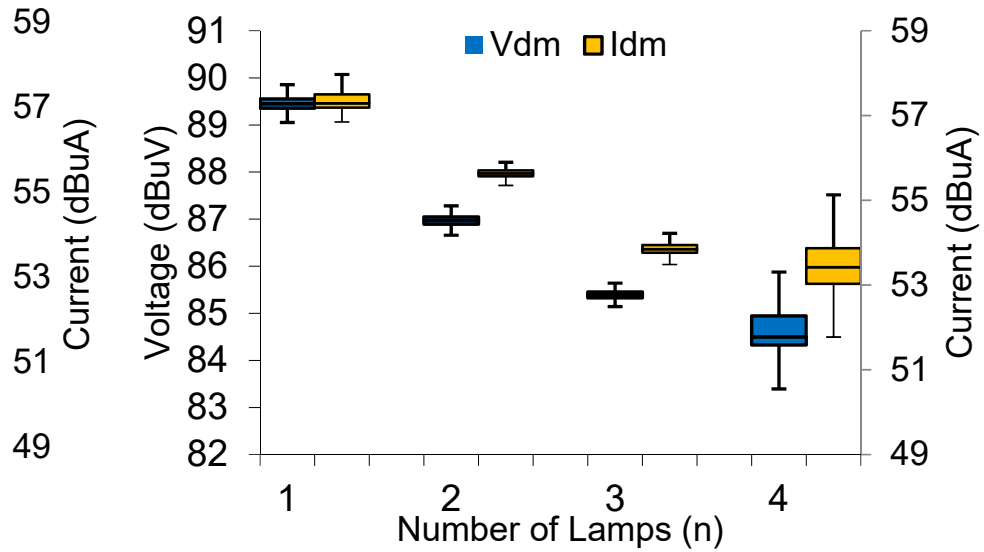

(d)

(e)

Figure 10. Box-and-whisky plots of AV detector for various topology. (a) Type-A connection. (b) Type-B connection. (c) Type-C connection. (d) Type-D connection. (e) Type-E connection.

The box-and-whisker results shown in Figure 10a-e correspond to the configurations depicted in Figure 9a-e. It can be seen the results obtained for both mean (median) $V_{\mathrm{DM}}$ and $I_{\mathrm{DM}}$ are having a declining trend with respect to higher number of CFL lamps. Generally, almost all the $V_{\mathrm{DM}}$ values obtained have a maximum gap about $4.5 \mathrm{~dB} \mu \mathrm{V}$ between one and four CFL lamps, their detrimental step between consecutive numbers of loads becomes narrower with respect to the increase of lamps connected. For instance, in Figure 10a, the mean value for one lamp is $89.4 \mathrm{~dB} \mu \mathrm{V}$, for two lamps it is $87.1 \mathrm{~dB} \mu \mathrm{V}$, for three lamps it is 
$85.1 \mathrm{~dB} \mu \mathrm{V}$ and four lamps it is $85 \mathrm{~dB} \mu \mathrm{V}$. Similar trend is seen in the $I_{\mathrm{DM}}$, but the magnitude of $I_{\mathrm{DM}}$ is slightly different for each configuration. The highest drop of $\mathrm{I}_{\mathrm{DM}}$ is measured for Figure 10e which drops from $57.6 \mathrm{~dB} \mu \mathrm{A}$ to $52 \mathrm{~dB} \mu \mathrm{A}$ corresponds to increasing number of lamps. On the other hand, the lowest $I_{\mathrm{DM}}$ value with the highest number of lamps connected is seen in the Figure $10 \mathrm{c}$ which is $50.2 \mathrm{~dB} \mu \mathrm{A}$. Furthermore, the mean peak DM values of these configurations are summarized in Figure 11 to compare their magnitude levels, it is obvious that different topology produces different EMI level with respects to the number of lamps. In these presented cases, it can be seen majority of measured aggregated mean EMI values with increasing number of lamps, their peak amplitudes of the $V_{\mathrm{DM}}$ and $I_{\mathrm{DM}}$ decrease instead of increase although some collected data might have adverse outcome due to inconsistent error in the outlier. Therefore, it is clear that for the presented grid configurations that contain multiple FL lamps, their contribution to the mean total peak emission particularly the DM voltage and DM current decrease due to the nature of these high-volatility sinusoidal components with large frequency step variation.

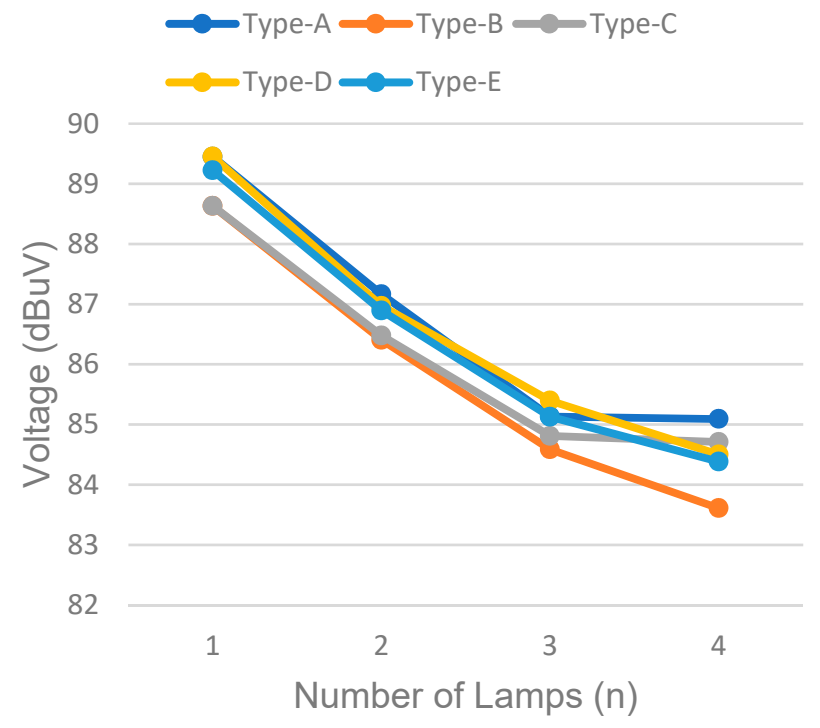

(a)

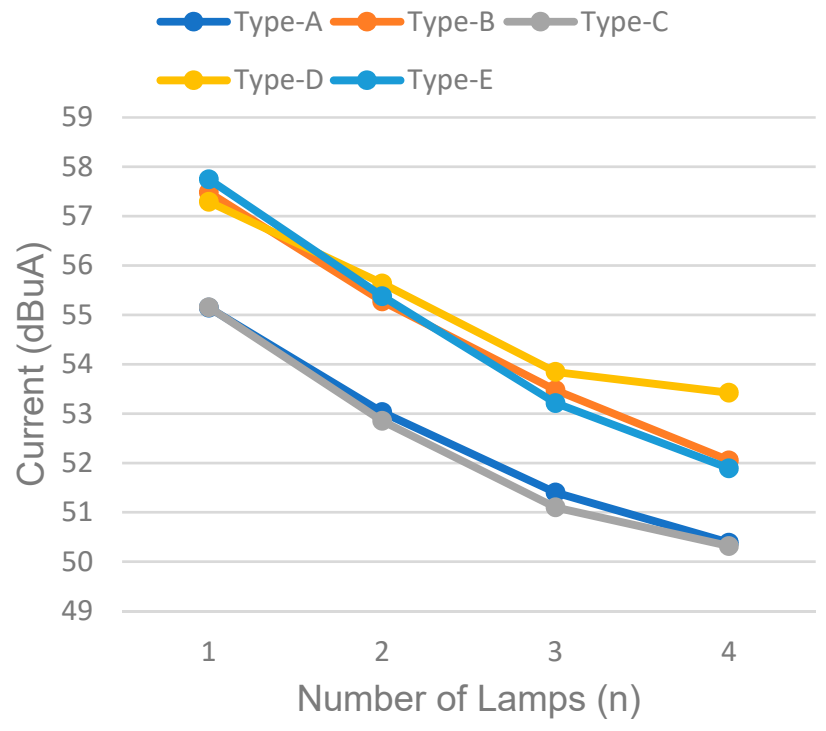

(b)

Figure 11. Line graph plots of mean peak DM conducted emission for various topology. (a) Voltage. (b) Current.

\section{Conclusions}

This paper has presented ostensible measurement outcome concerning highly volatile sinusoidal components. Furthermore, the concept is described and put to evaluate various grid topologies affecting the DM conducted emission emanating from CFL lamps. Results obtained have shown that the average aggregated DM conducted EMI decreases with the increase of FL lamps in grid. Further investigation of the effect of different topological network to the network impedance will be conducted.

Author Contributions: Conceptualization, methodology, writing—original draft, L.C.L.; writingreview and editing draft, investigation and validation, W.E.S.; writing-review and editing draft and formal analysis, V.M.; resources, writing-review and editing draft and supervision, N.M.; conceptualization and supervision, R.S.; resources and supervision, P.L. All authors have read and agreed to the published version of the manuscript.

Funding: This paper is part of a project that has received funding from the European Union's Horizon 2020 Research and Innovation Programme under the Marie Sklodowska-Curie Grant Agreement No. 812391-SCENT.

Conflicts of Interest: The authors declare no conflict of interest. 


\section{References}

1. European Commission. Commission Regulation (EC) No 244/2009 Directive 2005/32/EC of the European Parliament and of the Council with regard to ecodesign requirements for non-directional household lamps. Off. J. Eur. Union 2009, 47, 3-16.

2. European Commission. Commission Regulation (EC) No 245/2009 2005/32/EC with regard to ecodesign requirements for fluorescent lamps without integrated ballast, for high intensity discharge lamps, and for ballasts and luminaires able to operate such lamps, and repealing Directive 2000/55/EC of the European Parliament and of the Council. Off. J. Eur. Union 2009, 65, $17-44$.

3. IEA. Lighting; International Energy Agency: Paris, France, 2020.

4. Watson, R.; Hawkes, T.R.A.; Simbolotti, G. Lighting; IEA Energy Technology Systems Analysis Programme (ETSAP): Paris, France, 2012.

5. McGillicuddy, P.; De Grazio, D. Lighting Parameters for Building Information Modeling; Illuminating Engineering Society: New York, USA, 2019.

6. Hajjah Nor Maizura binti Yusoff. Guidelines on Occupational Safety and Health for Lighting at Workplace; Department of Occupational Safety and Health Malaysia: Putrajaya, Malaysia, 2018.

7. Yong, J.; Chen, L.; Nassif, A.B.; Xu, W. A Frequency-Domain Harmonic Model for Compact Fluorescent Lamps. IEEE Trans. Power Deliv. 2010, 25, 1182-1189. [CrossRef]

8. Nassif, A.B.; Xu, W. Characterizing the Harmonic Attenuation Effect of Compact Fluorescent Lamps. IEEE Trans. Power Deliv. 2009, 24, 1748-1749. [CrossRef]

9. Adebowale, E.; Shadare, M.S.; Musa, S.M. Electromagnetic compatibility issues in critical smart grid infrastructure. IEEE Electromagn. Compat. Mag. 2017, 6, 63-70.

10. Smolenski, R.; Kempski, A.; Benysek, G. Aggregated Conducted EMI Generated by Group of Frequency Converter-Fed Drives. In Proceedings of the 2009 IEEE Compatibility and Power Electronics, Badajoz, Spain, 20-22 May 2009; pp. 381-385.

11. Smolenski, R.; Lezynski, P.; Bojarski, J.; Drozdz, W.; Lok, C.L. Electromagnetic compatibility assessment in multiconverter power systems-Conducted interference issues. Meas. Elsevier 2020, 165, 108119. [CrossRef]

12. European Commission. Commission Regulation (EC) Directive 2014/30/EU on the Harmonisation of the Laws of the Member States Relating to Electromagnetic Compatibility, The European Parliament and the Council. Off. J. Eur. Union 2014, $96,79$.

13. Capponi, L.; Fernandez, I.; Roggo, D.; Arrinda, A.; Angulo, I.; De La Vega, D. Comparison of measurement methods of grid impedance for Narrow Band-PLC up to $500 \mathrm{kHz}$. In Proceedings of the 2018 IEEE 9th International Workshop on Applied Measurements for Power Systems (Amps), Aachen, Germany, 26-28 September 2018; pp. 82-87.

14. Emleh, A.; de Beer, A.S.; Ferreira, H.C.; Vinck, A.J.H. The impact of the CFL lamps on the power-line communications channel. The Impact of the CFL Lamps on the Power-Line Communications Channel. In Proceedings of the 2013 17th IEEE International Symposium on Power Line Communications and Its Applications (ISPLC), Johannesburg, South Africa, 24-27 March 2013; pp. 225-229.

15. Emleh, A.; De Beer, A.S.; Cheng, L.; Ferreira, H.; Vinck, A.H. An Overview of Colour LED \& CFL Lighting Interference on the Low Voltage PLC Network. In Proceedings of the 2017 40th International Conference on Telecommunications and Signal Processing (Tsp), Barcelona, Spain, 5-7 July 2017; pp. 220-224.

16. Castro, A.D.R.G.D.; Medina-Gracia, R.; Ronnberg, S.; Blanco, A.; Meyer, J. Differences in the performance between CFL and LED lamps under different voltage distortions. In Proceedings of the 2018 18th International Conference on Harmonics and Quality of Power (ICHQP), Ljubljana, Slovenia, 13-16 May 2018.

17. Lok, C.L.; Waseem, E.S.; Wibisono, M.A.; Moonen, N.; Smolenski, R.; Lezynski, P. Characteristic of Conducted EMI in Compact Fluorescent Lamps Application Assessment based on CISPR-11. In Proceedings of the Asia-Pacific International Symposium on Electromagnetic Compatibility (APEMC), Bali, Indonesia, 27-30 September 2021.

18. Ferreira, P.I.L.; Fontgalland, G.; Aragão, G.F.; Nascimento, A.R.Z.; Freire, R.C.S. Survey of Conducted EMI from Compact Fluorescent Lamps. Comput. Field Models Electromagn. Devices 2010, 34, 812-820.

19. Smolenski, R.; Kempski, A.; Bojarski, J.; Lezynski, P. EMI generated by Power Electronic Interfaces in Smart Grids. In Proceedings of the International Symposium on Electromagnetic Compatibility-EMC EUROPE, Rome, Italy, 17-21 September 2012.

20. Xie, D.G.; Hou, D.Y.; Fang, C.H.; Huang, M.L. On Simulation of Response Characteristic of EMI Test Receiver RF Front End. In Proceedings of the 2009 5th Asia-Pacific Conference on Environmental Electromagnetics, Piscataway, NJ, USA, 16-19 September 2009; pp. 164-167.

21. Hormaier, K.; Zangl, H.; Zojer, H. An EMI Receiver Model to Evaluate Electromagnetic Emissions by Simulation. In Proceedings of the 2012 IEEE International Instrumentation and Measurement Technology Conference (I2MTC), Graz, Austria, 13-16 May 2012; pp. 2558-2562.

22. Specification for Radio Disturbance and Immunity Measuring Apparatus and Methods-CISPR 16-1-1: Radio Disturbance and Immunity Measuring Apparatus_Measuring Apparatus; IEC: Geneva, Switzerland, 2015.

23. Pop, F.; Munteanu, C.; Răcăşan, A.; Păcurar, C.; Pruşu, S.; Mihai, G. Evaluation of Conducted Disturbances from LED Lamps According to EN 55015. In Proceedings of the 2016 International Conference on Communications (COMM 2016), Kuala Lumpur, Malaysia, 23-27 May 2016; pp. 509-512.

24. Aguilar, L.A.; Robledo-Sanchez, C.; Carrasco, M.A.; Otero, M.M. The principle of superposition for waves: The amplitude and phase modulation phenomena. Appl. Math. Inf. Sci. 2012, 6, 307-315. 
25. Li, C.; Zhang, L.; Dong, T.; Wang, T.; Chen, H. An EMI Receiver Model with Consideration of the Intermediate Frequency Filter. In Proceedings of the 2016 Asia-Pacific International Symposium on Electromagnetic Compatibility (APEMC), Shenzhen, China, 17-21 May 2016; pp. 233-236.

26. Zhang, X.; Zhao, Z.; Guo, F.; Meng, J.; Li, W.; Hu, A. Frequency Spectrum Analysis Method for Short-time and Frequency Conversion Signals Based on Simulation EMI Receiver. In Proceedings of the 2010 Asia-Pacific International Symposium on Electromagnetic Compatibility \& Technical Exhibition on Emc Rf/Microwave Measurements \& Instrumentation, Beijing, China, 12-16 April 2010; pp. 1606-1609.

27. Karaca, T.; Deutschmann, B.; Winkler, G. EMI-Receiver Simulation Model with Quasi-Peak Detector. In Proceedings of the 2015 IEEE International Symposium on Electromagnetic Compatibility (EMC), Dresden, Germany, 16-22 August 2015; pp. 891-896.

28. Braun, S.; Frech, A.; Russer, P. CISPR Specification and Measurement Uncertainty of the Time-domain EMI Measurement System. In Proceedings of the 2008 IEEE International Symposium on Electromagnetic Compatibility, Detroit Michigan, MI, USA, 18-22 August 2008; pp. 415-418.

29. Braun, S.; Donauer, T.; Russer, P. A Real-Time Time-Domain EMI Measurement System for Full-Compliance Measurements According to CISPR 16-1-1. IEEE Trans. Electromagn. Compat. 2008, 50, 259-267. [CrossRef]

30. Sarah Rönnberg, A.L. A Simple Model For Interaction Between Equipment At A Frequency Of Some Tens Of KHz. In Proceedings of the International Conference on Electricity Distribution, Frankfurt, Germany, 6-9 June 2011.

31. Lezynski, P.; Smolenski, R.; Loschi, H.; Thomas, D.; Moonen, N. A novel method for EMI evaluation in random modulated power electronic converters. Meas. Elsevier 2020, 151, 107098. [CrossRef]

32. Yang, Y.R. Comparison of Push-Pull and Half-bridge Resonant Inverters for Cold Cathode Fluorescent Lamps. In Proceedings of the 2015 IEEE 11th International Conference on Power Electronics and Drive Systems (PEDS 2015), Piscataway, NJ, USA, 9-12 June 2015; pp. 696-702.

33. Bayona, J.F.; Chamorro, H.R.; Sanchez, A.C.; Aguillon-Garcia, J.; Rubio, D.A. A High Efficiency Fluorescent Lamp Electronic Ballast Design. In Proceedings of the IEEE Cacidi 2016-IEEE Conference on Computer Sciences, Buenos Aires, Argentina, 30 November-2 December 2016.

34. Keyer, C.; Buesink, F.; Leferink, F. Mains Power Synchronous Conducted Noise Measurement in the 2 to $150 \mathrm{kHz}$ band. In Proceedings of the 2016 International Symposium on Electromagnetic Compatibility-EMC Europe, Wroclaw, Poland, 5-9 September 2016; pp. 865-869. 